\title{
On the Role of Gap Junctions in Cardiac Memory Effect
}

\author{
J Krishnan $^{1}$, VS Chakravarthy ${ }^{2}$, S Radhakrishnan ${ }^{1}$ \\ ${ }^{1}$ Department of Applied Mechanics, Indian Institute of Technology, Chennai, India \\ ${ }^{2}$ Department of Biotechnology, Indian Institute of Technology, Chennai, India
}

\begin{abstract}
Memory in the nervous system is essentially a network effect, resulting from synaptic adaptation in a network of neurons. The heart too is a network of cardiac cells electrically coupled by gap junctions, which, we hypothesize, adapt as a function of the state of cellular depolarization. Cardiac memory, which refers to persistent effect of external stimulus on activation pattern, is demonstrated in a pair of model Noble cells with adaptive gap junctions. The memory seems to have a dual effect: while it dissipates small perturbations, it captures and imprints larger ones. The proposed memory mechanism is also shown to increase likelihood of synchronization among cardiac cells. Finally we argue that the proposed mechanism is linked to known electrophysiological properties of gap junctions.
\end{abstract}

\section{Gap junction}

Gap Junctions (GJ) are one of the key means of cell-tocell communication for exchanging ions and small molecules between cells [1]. They are composed of oligomeric structures called connexons, which are constituted of a large family of protein subunits called connexins. A full GJ is formed of two connexons (there are hence called hemichannels) joined in series; each hemichannel spans the membrane of its respective cell. Connexin proteins are found in all vertebrates; invertebrates use a different family of proteins called innexins [1].

In humans there are at least 20 connexin genes expressed in a variety of systems. Defective connexin proteins are implicated a broad range of human diseases in cataractogenesis [2], hereditary deafness [3], infertility [4], Psoriasis [5], carcinogenesis [6] impaired inflammatory responses [7], X-linked Charcot-MarieTooth disease [1] etc. Particularly relevant to the present paper is the role of GJs in cardiac disease which have been related to heart failure [9], arrhythmogenesis [10], and atherosclerosis [11].

It is recognized that conduction properties of atria and ventricles depend on spatial distributions of GJs in the two regions [12]. Experimentally induced uncoupling of GJs resulted in altered activation sequence [13]. Abnormal GJ coupling is implicated as having a causative role in ventricular arrhythmias associated ischemic and hypertrophic heart diseases [10,14,15]. GJ distribution is also known to be remodeled by external pacing [16].

This crucial role of GJs in cardiac rhythm shaping is understandable if we adopt the view of heart as a network of cardiac oscillators communicating through conducting channels - the GJs. Since heart is a network, the pattern of connectivity determines activation sequence. Thus it is the GJs that primarily determine the network structure and electrical function of the heart. We have earlier suggested that the phenomenon of cardiac memory, which refers to the property of the heart to produce persistent responses to external stimuli, is closely related to GJs [17]. Our argument gains strength from the available knowledge of learning and memory mechanisms in nervous system.

\subsection{Neural memory mechanism}

A fundamental tenet of modern neuroscience is that synaptic modification underlies all learning and memory phenomena in the nervous system. Synaptic modification or plasiticity, is known to be activity-dependent, in that it depends on the electrical signaling in the neurons connected by the synapse. One such activity-dependent rule of synaptic plasticity is known as the Hebbian learning according to which a synapse connecting a pair of neurons with correlated activity must be strengthened; one connecting neurons with uncorrelated activity is weakened. Abundant evidence for Hebbian-like synaptic adaptation is found in the phenomena of LTP and LTD. The success of Hebbian mechanims in explaining memory mechanisms in the brain is well documented (see [15] for a survey).

\section{The model}

The Noble model [18], one of the most detailed biophysical models of cardiac cell, is used in our simulations. In abbreviated form the membrane voltage dynamics of Noble-Noble cell is given as: 


$$
C \frac{d V_{m}}{d t}=I^{\text {ionkin }}+I_{e x t}
$$

where $\mathrm{C}$ is the membrane capacitance, $\mathrm{V}_{\mathrm{m}}$ is the membrane voltage, $\mathrm{I}^{\text {ionkin }}$ represents all the currents flowing in due to kinetics of ion channels, $\mathrm{I}_{\mathrm{ext}}$ is the external current, if any. For more details of the model the reader may refer to original sources [18].

The Noble model is autorhythmic only for a range of values of leakage conductance (between 0.0068 and $3.45 \mathrm{e}-4 \mathrm{~S} / \mathrm{mm}^{2}$ ). When a pair of autorhythmic cells are coupled by a GJ conductance, $\mathrm{g}_{\mathrm{j}}$, voltage dynamics becomes:

$$
\begin{aligned}
& C \frac{d V_{1}}{d t}=I_{1}^{\text {ionkin }}+g_{j}\left(V_{2}-V_{1}\right) \\
& C \frac{d V_{2}}{d t}=I_{2}^{\text {ionkin }}+g_{j}\left(V_{1}-V_{2}\right)
\end{aligned}
$$

where $\mathrm{V}_{1}$ and $\mathrm{V}_{2}$ are the membrane voltages of cells 1 and 2 respectively; $\mathrm{I}_{1}{ }^{\text {ionkin }}$ and $\mathrm{I}_{2}{ }^{\text {ionkin }}$ represents the total ion channel currents in cells 1 and $2 ; \mathrm{g}_{\mathrm{j}}$ represents the conductance of the GJ connecting the two cells.

When the two cells $1 \& 2$ are in autorhythmic mode, they synchronize only when the individual frequencies of the two cells, $f_{1}$ and $f_{2}$ are sufficiently close, and the coupling conductance is sufficiently high. When the cells do synchronize, and beat at a common frequency, $f$ (where, $f_{1}<f<f_{2}$ ), the phase difference between the two cells, $\phi$, increases with decreasing conductance, $\mathrm{g}_{\mathrm{j}}$.

It has been proposed that cardiac memory gives increased stability to cardiac activation pattern in the sense that the memory mechanism can dissipate small perturbations to activity. Since a robust cardiac activation pattern depends on stable phase-relationships among cardiac oscillators GJs are most likely to have a role in the memory effect. Hence our hypothesis for GJ-based cardiac memory mechanism: cardiac memory is the result of a cell-to-cell interaction that tries to maintain a fixed relationship between phase and GJ coupling under varying external stimuli. Cells try to compensate for any change in phase difference by making adaptive changes to GJ coupling strength. This GJ adaptation dynamics is given as:

$$
\tau \frac{d w}{d t}=-w+\left(V_{1} V_{2}\right)
$$

where ' $w$ ' is an abstract variable representing short-term correlation between $\mathrm{V}_{1}(\mathrm{t})$ and $\mathrm{V}_{2}(\mathrm{t})$. Then, $\mathrm{g}_{\mathrm{j}}$ can be estimated from ' $w$ ' as:

$$
g_{j}=F(w),
$$

where for a given pair of cells, $\mathrm{F}(\mathrm{w})$ is found empirically from simulations. Eqn. (2.4) is analogous to Hebb-like mechanisms proposed to explain learning related phenomena in the brain.

\section{Simulation experiments}

All the simulations revolve around a pair of Noble cardiac cell models. Fig. 1 (a) shows the membrane voltages of two cells, with intrinsic frequencies $f_{1}=50$ bpm and $\mathrm{f}_{2}=60 \mathrm{bpm}$, and a coupling conductance $\left(\mathrm{G}_{\mathrm{cpl}}\right)$ of 5e-5 S/mm 2. A sharp spike followed by a plateau due to slow calcium kinetics, characteristic of cardiac action potential, can be seen in the waveform. External current inputs given to the two cells are generated by simulating another cell model with intrinsic frequency $\mathrm{f}=59 \mathrm{bpm}$. This frequency is chosen because it falls in the range of common frequencies at which the two cells entrain. The two external inputs have the same amplitude (15 $\mathrm{mA} / \mathrm{mm}^{2}$ ).

Since presentation of external inputs disturbs the relation between phase difference and coupling conductance, learning mechanisms (eqns. (2.4 \& 2.5)) are triggered and the cell pair system searches for a value of $\mathrm{G}_{\mathrm{cpl}}$ that is more appropriate for the new, imposed value of phase difference. For a cell pair (with $\mathrm{f}_{1}=50 \mathrm{bpm}$ and $\mathrm{f}_{2}=60 \mathrm{bpm}$ ) driven by external current inputs ( $\underline{\mathrm{I}}_{\underline{\mathrm{ext}}}=15$ $\mathrm{mA} / \mathrm{mm}^{2}$, phase difference, $\phi_{\mathrm{ext}}=45^{\circ}$ ) the phase difference between the membrane voltages of the two cells in four different stages -1) natural condition i.e., before learning without external input), 2) with external input, just before learning, 3) after learning with the external input still present, and 4) after learning with external input removed - are observed. The long term change in phase difference produced by the external input, supported by GJ adaptation, is the basic memory effect (Fig. 1).

In the second study, external inputs are given for both short (10 secs) and long duration (30 secs). In the case of short duration external signal, the effect of external signal disappears after removal of the signal, whereas in long duration signal, the cells search for a new value of $\mathrm{G}_{\mathrm{cpl}}$ and input signal disturbance is imprinted on the pair of cells (Fig. 2.)

The third study, two cells with a coupling conductance $\left(\mathrm{G}_{\mathrm{cpl}}=5 \mathrm{e}-5 \mathrm{~S} / \mathrm{mm}^{2}\right)$ entrain with a phase difference of $\phi=31.86^{\circ}$. This phase difference increases with decreasing $\mathrm{G}_{\mathrm{cpl}}$ (within limits). The range of values of $\mathrm{G}_{\mathrm{cpl}}$ for which a pair of cells are entrained depends on the difference in intrinsic frequencies of the two cells $\left(f_{1}-f_{2}\right)$ as well as coupling conductance. From our simulation, it is observed that the range of coupling conductance for which a pair of 


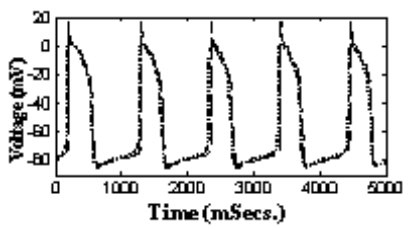

a Without $I_{\text {EXT }}$ before Le arning

$\mathrm{F}_{1}=60 \mathrm{bpth} \mathrm{F}_{2}=50 \mathrm{bpth}$

$F_{\text {commom }}=61$ bpom

$\phi=14.46^{\circ} \mathrm{G}_{\mathrm{CPL}}=5 \mathrm{e}-5 \mathrm{~S} / \mathrm{mm}^{2}$

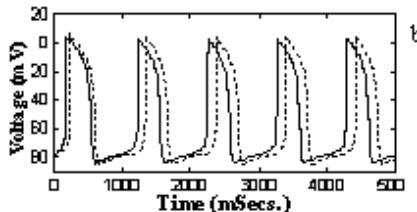

b. With $\mathrm{I}_{\mathrm{EXT}}$ before Lear ring $F_{1}=60$ bptm $F_{2}=50$ bpom

$\mathrm{F}_{\text {Common }}=61 \mathrm{bpth}$

$\phi=41.68^{\circ} \mathrm{I}_{\mathrm{EXT}}=15 \mathrm{~mA} / \mathrm{mm}^{2}$

$\phi_{\mathrm{ERT}}=45^{\circ} \mathrm{G}_{\mathrm{CPL}}=5 \mathrm{e}-5 \mathrm{~S} / \mathrm{mL}^{2} \mathrm{Lt}^{2}$

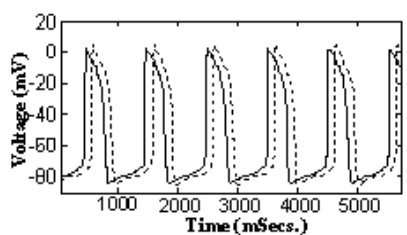

c. With $\mathrm{I}_{\mathrm{ERT}}$ after Learning

$F_{1}=60 \mathrm{bpm} \mathrm{F}_{2}=50 \mathrm{bpm}$

$\mathrm{F}_{\mathrm{Comm} m \mathrm{~m}}=61 \mathrm{bpum}$

$\phi F=52.811^{\circ} \mathrm{I}_{\mathrm{EST}}=15 \mathrm{mn} / \mathrm{mm} \mathrm{m}^{2}$

$\phi_{\mathrm{EXT}}=45^{\circ} \mathrm{G}_{\mathrm{CFI}}=4.432 \mathrm{e}-5 \mathrm{~s} / \mathrm{trtm}^{2}$

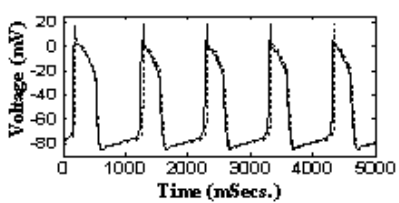

d. Without $\mathrm{I}_{\mathrm{EXT}}$ after Learriting

$\mathrm{F}_{1}=60 \mathrm{bpm} \mathrm{F}_{2}=50 \mathrm{bpm}$

$\mathrm{F}_{\mathrm{common}}=61 \mathrm{bpth}$

$\phi=19.08^{\circ} \mathrm{G}_{\mathrm{CPL}}=4.362 \mathrm{e}-5 \mathrm{~S} / \mathrm{mmm}^{2}$

Figure 1: Response of cell Pair with and without external signal and before and after learning.
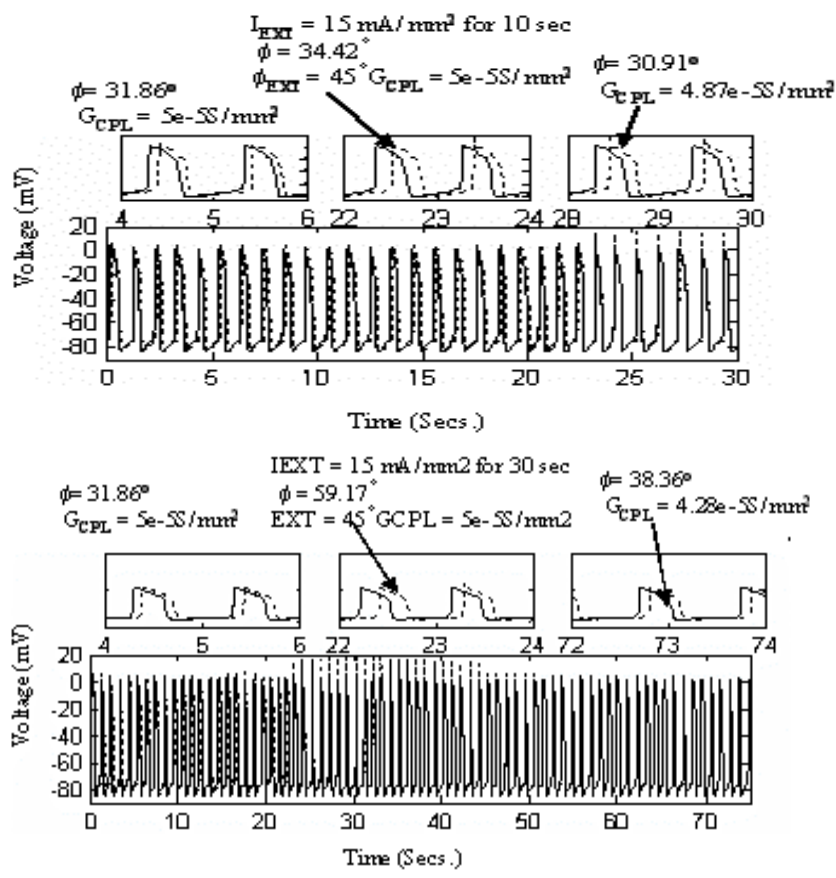

Figure 2: Response of cell pair with short and long duration external signal cells are entrained can be increased by the proposed GJ adaptation mechanism of eqn. 2.4 (Fig. 3). This shows that GJ adaptation improves synchronization.

In the fourth study, external inputs are presented with a frequency of 59 and variable phase difference $\left(0^{\circ}\right.$ to $150^{\circ}$ ). During this simulation, the steady state value of GJ conductance $\left(\mathrm{G}_{\mathrm{CPL}}(\mathrm{SS})\right)$ and $\mathrm{rms}$ value of junctional voltage (V_rms) have been recorded. The relation between $\mathrm{G}_{\mathrm{CPL}}(\mathrm{SS})$ and $\mathrm{V} \_$rms (Fig. 4) closely resembles the familiar bell-shaped relationship between $\left(\mathrm{G}_{\mathrm{CPL}}(\mathrm{SS})\right)$ and steady state junctional voltage $\left(\mathrm{V}_{\mathrm{j}}\right)$ [19].

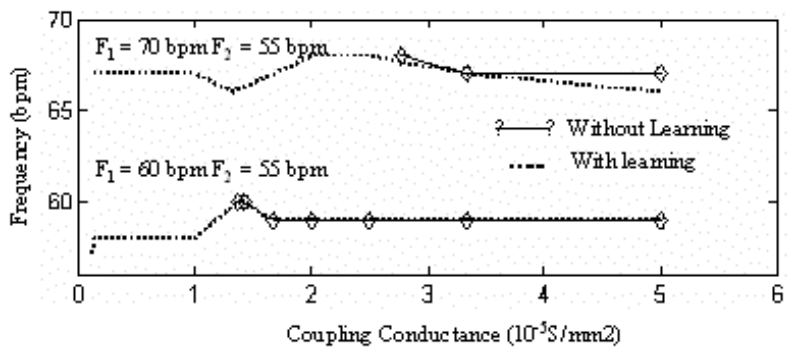

Figure 3: GJ adaptation increases synchronization in a pair of cells

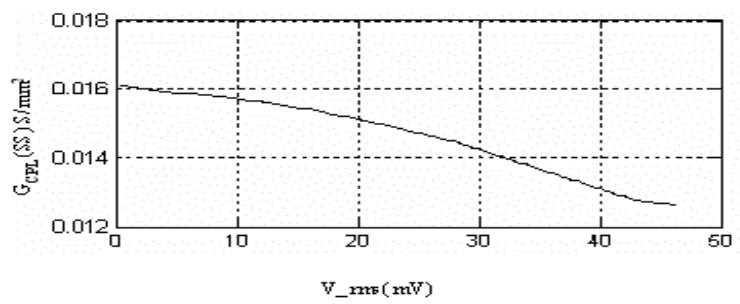

Figure 4: Bell-shaped relation between GJ conductance and rms Junctional voltage

\section{Discussion}

The paradigm shift that came in the field of neuroscience in the 80 's is often called "Connectionism" because it recognized the supreme importance of neural connections in determining brain function. It is now axiomatic that synaptic (connectionist) modification underlies learning and memory in the brain. Taking this analogy into cardiac electrophysiology we hypothesize that adaptation of GJs a la neurons forms the basis of cardiac memory effect [17].

In the present paper we show that the proposed mechanism of GJ adaptation explains several known phenomenological properties of cardiac memory. Most importantly we show that proposed GJ adaptation has deep ties with known electrophysiology of GJs - the bellshaped dependence of steady-state GJ conductance, $\mathrm{g}_{\mathrm{ss}}$ on 
junctional voltage, $\mathrm{V}_{\mathrm{j}}$. Our model points out the need to take a fresh look at the significance of GJ electrophysiological properties.

Standard protocols that study GJ electrophysiology involve a pair of coupled cells in which the voltage of one cells is clamped at a fixed value, while a step voltage is maintained (by feedback) in the second cell. The steady state GJ conductance has a bell-shaped dependence on junctional voltage $\mathrm{Vj}$, maintained at a constant level. It is not clear what is the relevance of such an experimental protocol to in vivo cardiac situation since in vivo cardiac activity is time-varying. Our simulations show that the same bell-shaped dependence is confirmed in oscillatory case also though the constant $\mathrm{Vj}$ is replaced by its RMS value. However, it remains to be seen experimentally whether GJ dynamics in cardiac cells interacting with action potentials follows the pattern of GJ evolution seen in currently applied protocols with steady state cell voltages.

\section{Acknowledgement}

We thank Department of Science and Technology, New Delhi for funding to carry out the research work.

\section{References}

[1] De Mello, W.C. Cell to cell communication in the failing heart, In Heart cell communication in health and disease. Boston.: Kluwer Academic Publishers, 1998: pp149-173.

[2] Gong, X., Li, E., Klier, G., Huang, Q., Wu, Y., Lei, H., Kumar, N.M., Horwitz, J. and Gilula, N.B. Disruption of alpha3 connexin gene leads to proteolysis and cataractogenesis in mice. Cell 1997; 91: 833-843.

[3] Kelsell, D.P., Dunlop, J., Stevens, H.P., Lench, N.J., Liang, J.N., Parry, G., Mueller, R.F. and Leigh, I.M. Connexin 26 mutations in hereditary non-syndromic sensorial deafness. Nature 1997; 387: 80-83.

[4] Simon, A.M., Goodenough, D.A., Li, E., Paul, D.L. Female infertility in mice lacking connexin 37. Nature 1997; 385:525-529.

[5] Labarthe, M.P., Bosco, D., Saurat, J.H., Meda, P., and Salomon, D. Upregulation of Connexin 26 betweem keratoinocytes of psoriatic lesions. J. Invest. Dermatol. 1998; 111: 72-76.

[6] Wilgenbaus, K.K., Kirkpatrick, C.J., Knuechel, R., Willecke, K., and Traub, O. Expression of Cx26, Cx32 and Cx43 gap junction proteins in normal and neoplastic human tissues. Int. J. Cancer 1992; 51: 522-529.

[7] Saez, J.C., Araya, R., Branes, M.C., Concha, M., Contreras, J.E., Eugenin, E.A., Martinez, A.D., Palisson, F. and Sepulveda, M.A. Gap Junctions in inflammatory responses: connexins, regulation and possible functional roles In "Gap Junctions: Molecular basis on cell communication in health and disease," C. Perrachia [Ed.], Current Topics in Membranes, Vol 49, Academic Press.
[8] Bone, L.J., Deschenes, S.M., Balice-Gordon, R.J., Fischbeck, K.H. and Scherer, S.S. Connexin32 and Xlinked Charcot-Marie-Tooth disease. Neurobiol. Dis. 1997; 4: 221-230.

[9] Peters NS. Myocardial gap junction organization in ischemia and infarction. Microsc Res Tech. 1995; 31:375386.

[10] Blackburn, J.P., Peters, N.S., Yeh, H.I., Rothery, S., Green, C.R., and Severs, N.J. Upregulation of connexin gap junctions during early stages of human coronary atherosclerosis. Arterioscler. Thromb. Vasc. Biol. 1995; 15:1219-1228.

[11] Saffitz JE. Gap junctions: functional effects of molecular structure and tissue distribution. Adv Exp Med Biol. 1997; 430:291-301.

[12] Dhein S, Krusemann K, Schaefer T. Effects of the gap junction uncoupler palmitoleic acid on the activation and repolarization wavefronts in isolated rabbit hearts. $\mathrm{Br} \mathrm{J}$ Pharmacol. 1999; 128:1375-84.

[13] Peters NS. New insights into myocardial arrhythmogenesis: distribution of gap-junctional coupling in normal, ischaemic and hypertrophied human hearts. Clin Sci. 1996; 90:447-452.

[14] Peters NS, Green CR, Poole-Wilson PA, Severs NJ. Reduced content of connexin43 gap junctions in ventricular myocardium from hypertrophied and ischemic human hearts. Circulation. 1993; 88:864-875.

[15] Patel PM, Plotnikov A, Kanagaratnam P, Shvilkin A, Sheehan CT, Xiong W, Danilo P Jr, Rosen MR, Peters NS. Altering ventricular activation remodels gap junction distribution in canine heart. Cardiovasc Electrophysiol.. 2001;12:570-577.

[16] Montague, P.R. and Sejnowski, T.J. The Predictive Brain: Temporal Coincidence and temporal order in Synaptic learning mechanisms. Learning \& Memory 1994 ; 1: 1-33.

[17] Chakravarthy, S. and Ghosh, J. On Hebbian-like Adaption in Heart Muscle: A proposal for "Cardiac Memory". Biological Cybernetics. 1997; 76: 207-215.

[18] Noble D. A Modification of the Hodgkin-Huxley Equations Applicable to Purkinje Fibre Action and Pacemaker Potentials. Jr. Physiology. 1960;160:317-352.

[19] Bennet MVL, Verselis VK. Biophysics of Gap Junctions. Sem.Cell. Biol. 1992; 3:29-47.

\author{
Address for Correspondance \\ Name : J.Krishnan \\ Address: \\ Research Scholar, Biomedical Engineering Division, \\ Department of Applied Mechanics, \\ Indian Institute of Technology, Madras. \\ Chennai - 600 036, India \\ Email : jkrishnan70@gmail.com \\ FAX : +91-44- 22574052
}

